\title{
Cosets and Ferromagnetic Correlation Inequalities
}

\author{
S. SHERMAN* \\ Department of Mathematics, Indiana University, Bloomington
}

Received May 3, 1969

\begin{abstract}
Consideration of subgroups of the group of all subsets of $N=\{1,2, \ldots, n\}$ with symmetric difference as the operation leads to new inequalities on correlations for a generalized Ising ferromagnet. An upper bound for the rate of change of $\left\langle\sigma^{R}\right\rangle$ with respect to $J_{S}$ in terms of correlations and a new, brief proof for the monotonicity of $\left\langle\sigma^{R}\right\rangle$ as a function of $J_{S}$ are given.
\end{abstract}

\section{§ 1. Introduction}

The success of Griffiths $[1,2]$ in establishing correlation inequalities (the non negativity of correlation and the monotonicity of correlation as a function of interaction) for Ising ferromagnets suggests the problem of getting other, if not all, correlation inequalities [3, Appendix, (1)] for generalized Ising ferromagnets.

In this paper other correlation inequalities are deduced as a consequence of considering a subgroup $\mathscr{G}_{0}$ of the group $\mathscr{G}=\left(2^{N}, \Delta\right)$ of subsets of $N=\{1,2, \ldots, n\}$, the set of spin locations, under the operation $\Delta$ of symmetric difference. In particular it is shown (in the notation of [3] which is used in the sequel) that

$$
\frac{\beta}{2}\left(1+\left\langle\sigma^{R} \sigma^{S}\right\rangle^{2}-\left\langle\sigma^{R}\right\rangle^{2}-\left\langle\sigma^{S}\right\rangle^{2}\right) \geqq \frac{d\left\langle\sigma^{R}\right\rangle}{d J_{S}} .
$$

A brief proof of the monotonicity of correlation as a function of interaction yields new correlation inequalities as a result of the need for using a sufficiently strong inductive hypothesis.

\section{$\S 2$. Cosets}

Let $\mathscr{G}={ }_{\text {df }}$ the group $\left(2^{N}, \Delta\right)$. Consider $J: \mathscr{G} \rightarrow R$ and $\mathscr{G}_{0} \prec \mathscr{G}\left(\mathscr{G}_{0}\right.$ a subgroup of $\mathscr{G})$. For all $A \in \mathscr{G}$, let

$$
\tilde{J}_{A \mathscr{G}_{0}}={ }_{\mathrm{df}} \sum_{B \in A \mathscr{G}_{0}} J_{B}
$$

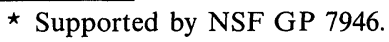

1 Commun. math. Phys., Vol. 14 
thus getting $\tilde{J}: \mathscr{G} / \mathscr{G}_{0} \rightarrow R$. The mapping $J \rightarrow \tilde{J}$ is a homomorphism of $L^{1}(\mathscr{G})$ into $L^{1}\left(\mathscr{G} / \mathscr{G}_{0}\right)$. If $\pi=\exp J$, in the real group algebra $\mathscr{A}(\mathscr{G})$, then

in $\mathscr{A}\left(\mathscr{G} / \mathscr{G}_{0}\right)$.

$$
\exp \tilde{J}=\tilde{\pi}=\sum_{D \in \mathscr{G} / \mathscr{G}_{0}} \tilde{\pi}_{D} D
$$

Special case: $\mathscr{G}_{0}=\{\phi, R\}$

$$
\begin{aligned}
\tilde{\pi}_{\mathscr{G}_{0}} & =\pi_{\phi}+\pi_{R}, \\
\tilde{\pi}_{S \mathscr{G}_{0}} & =\pi_{S}+\pi_{R S} .
\end{aligned}
$$

$J$ ferromagnetic, i.e., $(\forall A \neq \phi) \quad J_{A} \geqq 0: \Rightarrow: \tilde{J}$ ferromagnetic. $J$ ferromagnetic $\Rightarrow \pi_{\phi}+\pi_{R} \geqq \pi_{S}+\pi_{R S}$, since $\tilde{\pi}_{\mathscr{G}_{0}} \geqq \tilde{\pi}_{S \mathscr{G}_{0}}$.

Theorem 1. $\frac{\beta}{2}\left(1+\left\langle\sigma^{R} \sigma^{S}\right\rangle^{2}-\left\langle\sigma^{R}\right\rangle^{2}-\left\langle\sigma^{S}\right\rangle^{2}\right) \geqq \frac{d\left\langle\sigma^{R}\right\rangle}{d J_{S}}$.

Proof. From $\pi_{\phi}+\pi_{R} \geqq \pi_{S}+\pi_{R S}$ it follows $\pi_{\phi}-\pi_{R S} \geqq \pi_{S}-\pi_{R}$. From $\pi_{\phi}+\pi_{S} \geqq \pi_{R}+\pi_{R S}$ it follows $\pi_{\phi}-\pi_{R S} \geqq \pi_{R}-\pi_{S}$. Thus

$$
\left(\pi_{\phi}-\pi_{R S}\right)^{2} \geqq\left(\pi_{S}-\pi_{R}\right)^{2} \text { and } \pi_{\phi}^{2}+\pi_{R S}^{2}-\pi_{R}^{2}-\pi_{S}^{2} \geqq 2 \pi_{\phi} \pi_{R S}-2 \pi_{R} \pi_{S} \text {. }
$$

\section{§ 3. Monotonicity of Correlation}

For $\pi=\exp J$,

and

$$
\frac{d \pi}{d J_{D}}=\pi D
$$

$$
\frac{d \pi_{A}}{d J_{D}}=\pi_{A D} .
$$

Let $\delta\left(\mathscr{G}_{0} ; R, S\right)={ }_{\mathrm{df}} \sum_{B \in \mathscr{C}_{0}}\left(\pi_{B} \pi_{B R}-\pi_{B S} \pi_{B R S}\right)$.

Theorem 2. $J$ ferromagnetic $\Rightarrow a$ ) $\delta \geqq 0$, and b) $\frac{d \delta}{d J_{D}} \geqq 0$.

Proof. Fix $N$ and let $P={ }_{\mathrm{df}}\left\{D: J_{D}>0\right\}$. Proceed by induction on \# $P$. If $\# P=0$, then

and

$$
B \neq \phi \Rightarrow \pi_{B}=0
$$

$$
B=\phi \Rightarrow \pi_{B}>0 .
$$

Since $\mathscr{G}_{0}$ is a group, $\phi \in \mathscr{G}_{0}$. For $R=\phi$.

$$
\sum_{B \in \mathscr{G}_{0}} \pi_{B} \pi_{B R}=\pi_{\phi}^{2}
$$


and

$$
0 \leqq \sum_{B \in \mathscr{G}_{0}} \pi_{B S} \pi_{B R S} \leqq \pi_{\phi}^{2},
$$

so $\delta\left(\mathscr{G}_{0} ; R, S\right) \geqq 0$. For $R \neq \phi, \delta\left(\mathscr{G}_{0} ; R, S\right)=0$. Thus (a) holds if \#P=0. Suppose (a) holds $\left(\forall \mathscr{G}_{0} \prec \mathscr{G}\right)(\forall R, S \in \mathscr{G})$ if $\# P \leqq k$. Consider \#P=k+1. Note that

$$
\begin{aligned}
\frac{d \delta\left(\mathscr{G}_{0} ; R, S\right)}{d J_{D}} & =\sum_{B \in \mathscr{G}_{0}} \pi_{B D} \pi_{B R}+\pi_{B} \pi_{B R D}-\pi_{B S D} \pi_{B R S}-\pi_{B S} \pi_{B R S D} \\
& = \begin{cases}\delta\left(\left[\mathscr{G}_{0} \cup\{D\}\right] ; R D, S\right), & D \notin \mathscr{G}_{0}, \\
2 \delta\left(\mathscr{G}_{0} ; R D, S\right), & D \in \mathscr{G}_{0}\end{cases}
\end{aligned}
$$

where $[\mathscr{D}]$ is group generated by the elements of $\mathscr{D}$. Suppose $D \in P$.

First consider $D \in \mathscr{G}_{0}$, the possible behavior of $\delta$, where the last argument is $J_{D}$, is given by the following table:

Table

\begin{tabular}{lll}
\hline & $\delta\left(\mathscr{G}_{0} ; R, S ; 0\right)$ & $\delta\left(\mathscr{G}_{0} ; R D, S ; 0\right)$ \\
\hline$(1)$ & 0 & 0 \\
$(2)$ & + & 0 \\
$(3)$ & 0 & + \\
$(4)$ & + & + \\
\hline
\end{tabular}

In case $(1),\left(\forall J_{D} \geqq 0\right) \delta\left(\mathscr{G}_{0} ; R, S ; J_{D}\right) \equiv \delta\left(\mathscr{G}_{0} ; R D, S ; J_{D}\right) \equiv 0$. In cases $(2-4),\left(\forall J_{D}>0\right) \delta\left(\mathscr{G}_{0} ; R, S ; \mathrm{J}_{D}\right)>0$ and $\delta\left(\mathscr{G}_{0} ; R D, S ; J_{D}\right)>0$.

Next consider $D \notin \mathscr{G}_{0}$. From $D \in\left[\mathscr{G}_{0} \cup\{D\}\right]$ and the inductive hypothesis, $\left(\forall J_{D} \geqq 0\right) \delta\left(\mathscr{G}_{0} ; \omega, S ; J_{D}\right) \geqq 0$. Thus (a) holds $(\forall P)$ and from the expression for the derivative of $\delta$ with respect to $J_{D},(\mathrm{~b})$ holds $(\forall P)$. The proof is complete.

Corollary. $J$ ferromagnetic,

$$
\mathscr{G}_{0}\left\langle\mathscr{G} \Rightarrow \sum_{B \in \mathscr{G}_{0}}\left(\left\langle\sigma^{B}\right\rangle\left\langle\sigma^{B R}\right\rangle-\left\langle\sigma^{B S}\right\rangle\left\langle\sigma^{B R S}\right\rangle\right) \geqq 0 .\right.
$$

Special case: $\mathscr{G}_{0}=\{\phi\}$. Here the inequality becomes

$$
\left\langle\sigma^{R}\right\rangle-\left\langle\sigma^{S}\right\rangle\left\langle\sigma^{R S}\right\rangle \geqq 0
$$

which implies that the correlations are monotone functions of interactions in a generalized ferromagnetic Ising model.

Added in proof. Professor J. Ginibre has an elegant proof of Griffith's second theorem as well as an elegant generalization of Theorem 2 of this paper. These will appear in the lecture notes of the 1969 Cargese NATO Summer School in Theoretical Physics. 


\section{References}

1. Griffiths, R. B.: J. Math. Phys. 8, 478 (1967).

2. - J. Math. Phys. 8, 484 (1967).

3. Kelly, D. G., and S. Sherman: J. Math. Phys. 9, 466 (1968).

\section{S. Sherman} Indiana University Department of Mathematics Swain Hall-East

Bloomington, Indiana 47401, USA 